\title{
ARTICLE \\ High-Resolution Radiometer for Remote Sensing of Solar Flare Activity from Low Earth Orbit Satellites
}

\author{
Luca Aluigi* \\ DISMI, University of Modena and Reggio Emilia, Reggio Emilia, Italy
}

\section{ARTICLE INFO}

Article history:

Received: 20 December 2018

Accepted: 4 January 2019

Published: 4 January 2019

Keywords:

Dicke radiometer

Heliosynchronous orbit

Ionosphere

Microelectronics

MMIC

mm-waves

Sensor

Sun.

\section{ABSTRACT}

Solar flares, intense bursts of radiation, can disrupt the atmosphere and potentially affect communication, navigation and electrical systems. A newly developed miniaturised microwave radiometer used on a space-borne platform should offer astronomers unprecedented understanding of the largest explosive phenomena in our solar system. In this paper the activity and results of the EU funded research project FLARES are presented. Objective of FLARES has been the study, analysis and design of millimetre-wave system-on-chip (SoC) radiometers for space-borne detection of solar flares. Thanks to the proposed methodology, the power consumption and encumbrance (volume, weight) of the instrument can be sensibly reduced when compared to the devices currently in operation for observing and studying solar flares. In particular, the proposed SoC Dicke radiometer can achieve a ten-time better resolution. This in turn, allows detecting solar flares having relatively low intensity, about 100 times lower when compared to the flares currently detected by the existing systems, owing to space-borne operations and the microchip-level miniaturization through silicon technology under space qualification.

\section{Introduction}

$\times$

$\mathrm{R}$ ecently there has been an increase in media coverage bringing the focus on how our Sun can have an impact on technologies. This includes the Space Weather and its effects, which are now increasingly understood and taken into deeper consideration by the scientific community, the politicians and the public. Space Weather (SW) refers to the dynamic conditions in the
Earth's outer space environment, including conditions and events on the Sun, that can impact orbiting and terrestrial systems and through these, human life.

Conditions and events on the Sun contribute to SW with a number of different emission mechanisms. Here, we focus on the emission from solar flares.

Solar flares are a sudden release of a large amount of energy stored as high magnetic fields in the solar active

*Corresponding Author:

Luca Aluigi

DISMI, University of Modena and Reggio Emilia, Reggio Emilia, Italy

Email:luca.aluigi@unimore.it 
regions. During solar flares there is a large increase (burst) in emission lasting minutes to hours. The bursts originate from bremsstrahlung (accelerated electrons due to collisions with ions), gyro-synchrotron (accelerated electrons spiralling in magnetic fields), and plasma radiations. The released magnetic energy is rapidly converted into thermal, kinetic and mechanical energies and the consequence is that the local plasma is heated up to several ten of million degrees, while particles are accelerated up to high energies. Characteristics of these bursts due to solar flares vary with wavelength and the range of wavelengths at which they radiate goes from radio wave, soft and hard-X rays, up to $\gamma$-rays with energies reaching $1 \mathrm{Gev}^{[1]}$.

Flares interact with Earth's magnetic field and cause geomagnetic disturbances (GMDs). These in turn cause geo-magnetically induced currents (GICs) which can damage the electric power systems (e.g. damage to high-voltage transformers, loss of reactive power support) ${ }^{[2]}$.

Flares have a strong impact on Earth's ionosphere, causing an alteration of the electron density profile, also called Sudden Ionospheric Disturbance (SID). Such changes in the ionosphere can interfere with wireless systems and GNSS receivers ${ }^{[3]}$. Robotic and human explorers of the solar system can also be affected by solar activity, and particularly by flares and Coronal Mass Ejections (CMEs). Ionospheric disturbances can also affect critical phases of the Earth re-entry of spacecrafts by altering the calculation of the solutions of navigation trajectory ${ }^{[4]}$.

In this paper, we focus on the radio wave component of solar flare spectral emission, and particularly on the millimetre-wave (mm-wave) portion in the Ka-band, which is closely linked to many of the events triggered by flares, e.g. the connection between $\gamma$-ray emissions and $37 \mathrm{GHz}$ flares ${ }^{[5]}$. In particular, our study was developed in the frame of the European project FLARES ${ }^{[6]}$ aimed to the SoC implementation of $\mathrm{mm}$-wave Dicke radiometer for detection of solar flares from an orbiting system.

Millimetre-wave radiometers are commonly employed in scientific radio astronomic imaging experiments ${ }^{[7]}$. They are among the most sensitive systems currently available to observe the highest energy electrons accelerated in solar flares ${ }^{[8]}$. Dedicated spatially unresolved spectroscopic observations of the Sun at mm-waves are used in order to obtain the peak- and high-frequency slope of radio spectra, both used as a diagnostic tool for energy release and transport. Observations of multiple mm-wavelengths are also exploited for dynamic spectra analysis of prolonged emissions, and to study solar CMEs, which represent the main phenomena at the basis of $\mathrm{SW}^{[9]}$.

Large efforts have been spent to implement high stability ${ }^{[10]}$, accurate ${ }^{[11]}$ and compact ${ }^{[12]}$ micro-/mm-wave radiometers. These instruments were implemented in hybrid technologies and did not consider encumbrance constraints. One of the first attempts towards miniaturization has been presented in $2007^{[13]}$.

Space-borne mm-wave radiometers are commonly realized using hybrid technology processes ${ }^{[14]}$, thereby resulting bulky, heavy and expensive. Space agencies and research bodies are constantly scouting technological solutions that are able to mitigate the stringent encumbrance constraints imposed by micro-, nano-, and pico-satellites ${ }^{[15,16]}$. This translates into the need of having miniaturized instruments. The $\mathrm{SoC}$ integration through $\mathrm{SiGe}$ BiCMOS technology is probably the most efficient mean of meeting the miniaturization requirement ${ }^{[17]}$. The SiGe technology in fact, despite having slower transistors compared to III-V technologies, provides dramatic benefits in terms of yield, cost, miniaturization, and possibility of co-integration with other analog and digital circuitry on the same silicon die.

The first works investigating the SoC integration of radiometers in the microwave range appeared in $2008^{[18,19]}$ and in the mm-wave range in $2010^{[20,21]}$. Since then, several research projects and groups worldwide have been focusing on this attractive challenge with outstanding results ${ }^{[22]}$. However, the first works were carried out in latest silicon technology nodes and were addressed to terrestrial applications. To our best knowledge, no works have been addressed to space applications, even less through suitable space-qualified technologies. Moreover, thanks to the SoC miniaturization, many new research directions are opened up, which could enhance the effectiveness of the proposed methodology. For instance, in ${ }^{[23]}$ the near-field power transfer from chip to package applied to mm-wave antennas and SoC radiometers is presented. This approach can contribute to the optimization of the system.

In ${ }^{[24,25]}$ we carried out for the first time some preliminary investigations about the opportunity to implement SoC radiometers for space-borne observations of solar flares.

Following the contents in our previous conference communications ${ }^{[25-27]}$, in this paper we go beyond and sum up the results of FLARES ${ }^{[6]}$. Section 2 introduces to the methodology of the observation of solar flares in the Ka-band frequency range. Ground- and space-based observations are compared and the resolution equation of a balanced Dicke radiometer for solar flares observations is presented. Section 3 presents a summary of the results obtained for the simulations of the most important building blocks of the radiometer, namely the detector and the lownoise amplifier (LNA). Section 4 reports the discussion of the results and the conclusions are given in Section 5. 


\section{Methodology}

\subsection{Observations of Solar Flares in the Ka-band Frequency Range}

In radio astronomy, emission from the Sun captured by telescopes is measured in terms of spectral flux density $\mathrm{Sf}$, expressed in solar flux units (sfu). One sfu is equal to $10^{-22}$ $\mathrm{W} \mathrm{m}{ }^{-2} \mathrm{~Hz}^{-1}$. Measurements of the solar flux density at Kaband are available from the NORP and Metsahövi groundbased instruments ${ }^{[28,29]}$. The list of flares measured from the ground at mm-waves is sensibly reduced because the detection is limited by random fluctuations of the ionospheric transmissivity, leading to errors up to $\Delta \mathrm{S}_{\text {ferr }} \approx 200$ $\mathrm{sfu}^{[30]}$. Moreover, the night-day cycle limits the time-ontarget to an average of 12 hours per day, in good weather conditions (i.e. best case).

To achieve a drastic reduction of errors probability, thus improving the quantity of detected mm-wave flares, the radiometric sensor should be moved above the troposphere, e.g. in the ionosphere. Moreover, to carry out 24 hour time-on-target observations, it should be orbiting in Heliosynchronous low Earth orbit (Fig. 1).

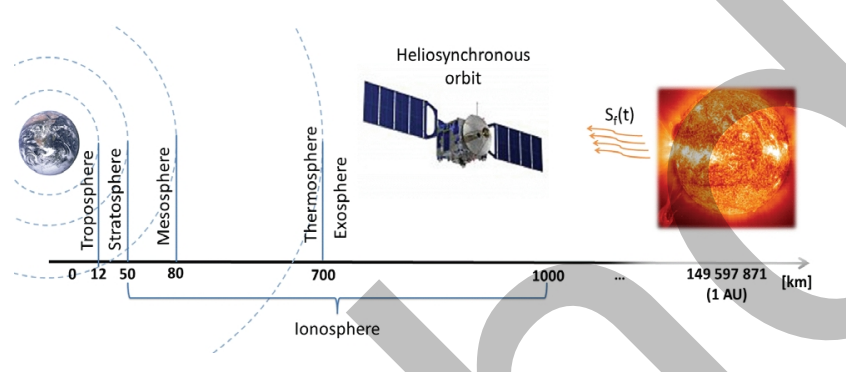

Figure 1. Heliosynchronous low Earth orbit

An Heliosynchronous ionospheric orbiting satellite is considered as housing platform for the radiometric sensor. By realizing this concept, it is possible to drastically reduce the impact of the fluctuations of the ionosphere with respect to ground-based instruments. Moreover, the instrument would be able to carry out 24 hour time-ontarget observations.

The ionosphere consists of electrons and electrically charged atoms and molecules. In the magneto-ionic theory, ionosphere is modeled as stratified plasma ${ }^{[31]}$. The absorption depends on electron density and collision frequency, which depend on height from Earth's surface. Based on the radiative transfer theory ${ }^{[32]}$ and considering a worst-case scenario, we can calculate the lower absolute bound of transmissivity $(\mathrm{T})$ in the ionosphere by exploiting the data for electron density and collision frequency in ${ }^{[33]}$, and considering an orbit height of $500 \mathrm{~km}$ above Earth's surface. The resulting equation for $\mathrm{T}$ is
$\mathrm{T}=e^{-\int_{0}^{\infty} \alpha(x) d x}$

where $\alpha(\mathrm{x})$ is the radio wave attenuation constant, which is a function of the position of the satellite with respect to Earth's surface, and proportional to the plasma conductivity ${ }^{[34]}$. Details about the derivation of (1) can be found in ${ }^{[30]}$. As shown in Fig. 2, the absorption (1-T) ranges from $48.3 \mathrm{ppm}$ at $26 \mathrm{GHz}$ to $20.4 \mathrm{ppm}$ at $40 \mathrm{GHz}$. The absorption caused by the ionospheric variability at the center frequency $\mathrm{f}_{0}=36.8 \mathrm{GHz}$ is $24.14 \mathrm{ppm}$, which leads to an error $\Delta \mathrm{S}_{\text {ferr }}=0.053 \mathrm{sfu}$.

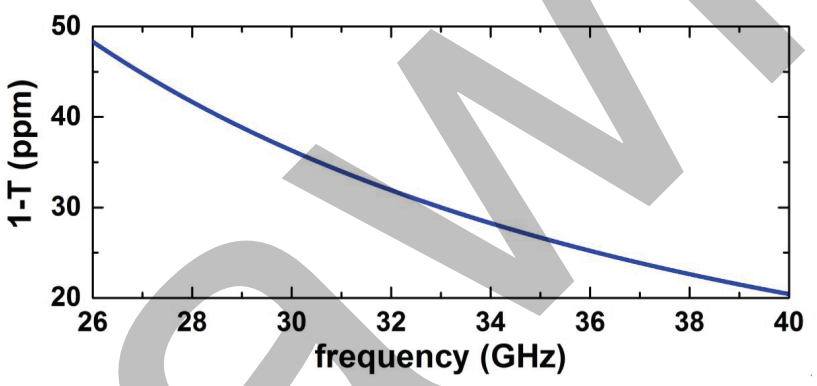

Figure 2. Ka-band absorption (1-T) frequency

Ka-band absorption (1-T) of the ionosphere from a satellite when the radio path is oriented towards the Sun starting from a satellite in LEO orbit at $500 \mathrm{~km}$ above Earth's ground.

\subsection{Dicke Radiometer for Solar Flares Observation}

Fig. 3 shows the block diagram of the proposed SoC Dicke radiometer. The Dicke architecture ${ }^{[35]}$ minimizes the variations in the system gain, i.e. the $\Delta \mathrm{G} / \mathrm{G}$ effect, which can severely affect basic radiometers such as the total power radiometer (TPR). The Dicke architecture is the most used and effective improvement to the TPR. As shown in Fig. 3, the input is switched periodically with frequency $\mathrm{f}_{\mathrm{S}}$ and duty cycle $\delta$ between the antenna at temperature $T_{A}$ and the reference noise source at temperature $\mathrm{T}_{\mathrm{N}}$, while at the same time, the output is synchronously demodulated by multiplying the detected signal by \pm 1 before the integrator.

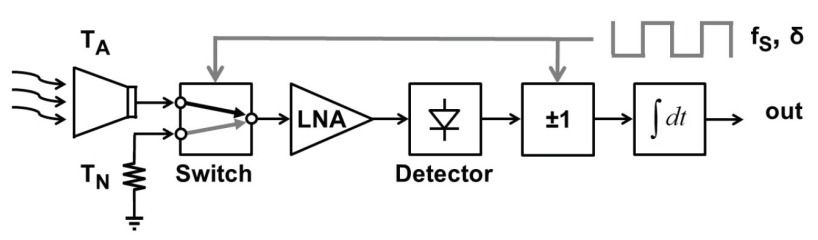

Figure 3. Architecture of the proposed radiometer employing a Dicke's switching configuration

The temperature resolution $\rho_{\mathrm{T}}$, commonly referred to as noise-equivalent delta temperature $(\mathrm{NE} \Delta \mathrm{T})$, is expressed as ${ }^{[36]}$ : 


$$
\rho_{T}=\sqrt{\frac{2\left(T_{A}+T_{R}\right)^{2}}{B W \tau}+\frac{2\left(T_{N}+T_{R}\right)^{2}}{B W \tau}+2\left(T_{A}-T_{N}\right)^{2}\left(\frac{\Delta G}{G}\right)^{2}}
$$

where $T_{R}=(F-1) T_{0}$ is the receiver equivalent noise temperature; $\mathrm{F}$ is the receiver noise factor and $\mathrm{T}_{0}$ is the standard IEEE temperature (i.e. $290 \mathrm{~K}$ ); BW is the system bandwidth; $\tau$ is the integration time; $\Delta \mathrm{G} / \mathrm{G}$ represent the variations in the system gain and is expressed in ppm. As can be deduced from (2), the effect of gain fluctuation can be highly mitigated by imposing $\mathrm{T}_{\mathrm{N}} \approx \mathrm{T}_{\mathrm{A}}$ and can ideally be eliminated if $\mathrm{T}_{\mathrm{N}}=\mathrm{T}_{\mathrm{A}}$. When the latter condition is met the Dicke radiometer is balanced by definition. Without loss of generality, we will assume here that the radiometer is balanced ${ }^{[25]}$.

In solar radio astronomy, $(2)$ is expressed in terms of $\left(\mathrm{S}_{\mathrm{f}}\right)$ as follows ${ }^{[24]}$ :

$$
\rho_{s f u}=2 \mathrm{~S}_{\mathrm{f}}\left(1+\frac{\mathrm{T}_{\mathrm{R}}}{\mathrm{T}_{\mathrm{A}}}\right) \sqrt{\frac{1}{\mathrm{BW} \tau}+\left[\frac{\mathrm{NEP}}{\mathrm{k}_{\mathrm{B}}\left(\mathrm{T}_{\mathrm{A}}+\mathrm{T}_{\mathrm{R}}\right) \mathrm{BWG} \sqrt{2 \tau}}\right]^{2}}
$$

where $\mathrm{k}_{\mathrm{B}}$ is Boltzmann constant, $\mathrm{G}$ is gain, NEP is the noise equivalent power of the detector defined as

$$
N E P=\frac{v_{n}\left(f_{S}\right)}{\mathfrak{R}}
$$

where $\operatorname{vn}\left(\mathrm{f}_{\mathrm{S}}\right)$ is the output noise of the detector at $f_{S}$ (expressed in $\mathrm{V} / \sqrt{ } \mathrm{Hz}$ ), and is the responsivity defined as the ratio of output voltage over RF input power

$$
\Re=\frac{V_{\text {out }}}{P_{\text {in }}}
$$

The two advantages of the Dicke balanced radiometer are that 1) the $\Delta \mathrm{G} / \mathrm{G}$ effect is removed, and 2) the flicker noise at the receiver output is modulated around $\pm \mathrm{f}_{\mathrm{S}}$ thus drastically reducing the noise within the integrator bandwidth. As a downside, the two disadvantages are that 1) the observation time is only half of the operating time, and 2 ) the switch causes a reduction of the system gain and an increment of the noise figure ${ }^{[37]}$.

The proposed SoC Dicke radiometer works at $\mathrm{f}_{0}=36.8$ $\mathrm{GHz}$, and targets a resolution $\rho_{\text {sfu }}=1 \mathrm{sfu}$ corresponding to a ten-time improvement with respect to state-of-the-art radiometers for the observation of solar flares ${ }^{[28,29]}$. The switching frequency $\left(\mathrm{f}_{\mathrm{S}}\right)$ of the radiometer is set to $1 \mathrm{MHz}^{[25]}$.

\section{Results}

To estimate the achievable resolution, with reference to Fig. 3 we consider the design of the key building blocks, namely the LNA and the detector.

The adopted technology is the SG13S/RH 130nm SiGe BiCMOS by IHP Microelectronics. The technology is currently being space qualified by the European Space Agency (ESA) and by the DLR (German Aerospace Center) ${ }^{[38]}$. This process allows for seven metal layers, npn heterojunction bipolar transistors (HBT) having a maximum transition frequency $\left(\mathrm{f}_{\mathrm{t}}\right)$ of about $250 \mathrm{GHz}$ and maximum oscillation frequency $\left(f_{\max }\right)$ of about $340 \mathrm{GHz}$. For the circuit simulations ADS by Keysight has been used, and in particular transient, s-parameter and Harmonic Balance simulations have been exploited. The spiral inductors have been synthesized using the automatic design synthesis methodology in ${ }^{[39]}$.

\subsection{Detector}

Exploiting the results in ${ }^{[26]}$, we address here the design of a common-emitter (CE) detector to be employed for the implementation of the proposed SoC Dicke radiometer introduced in the previous section. The goal of the designed detector is to ensure the best trade-off in terms of high, low NEP and the lowest possible power consumption $\left(\mathrm{P}_{\mathrm{C}}\right)$. A high allows relaxing the effective number of bits (ENOB) specification for the analog-to-digital converter (ADC). A low NEP corresponds to extending the sensitivity of the detector, i.e. capability to detect input signals with lower power levels, so relaxing the gain specification of the low noise amplifier (LNA).

The detector features a symmetric dummy circuit. The dummy circuit provides a reference dc output voltage $\left(\mathrm{V}_{\text {out- }}\right)$ for the output signal $\left(\mathrm{V}_{\text {out }}\right)$, so that $\mathrm{V}_{\text {out }}=\mathrm{V}_{\text {out }}-\mathrm{V}_{\text {out- }}$. The design has been carried out in order to exploit the following large-signal exponential characteristic of the transistors in the forward active region:

$$
I_{C}=I_{S} e^{V_{B E} / V_{T}}
$$

where $I_{C}$ is the collector current $\left(I_{C}=A_{E} \times J_{C}\right)$, IS is the reverse saturation current, $\mathrm{V}_{\mathrm{BE}}$ is base-emitter voltage and $\mathrm{V}_{\mathrm{T}}$ is the thermal voltage. The value of $\mathrm{J}_{\mathrm{C}}$ is selected in correspondence of the bias region with the higher dc current gain $\mathrm{I}_{\mathrm{C}} / \mathrm{I}_{\mathrm{B}}\left(\beta_{\mathrm{DC}}\right)$ of the transistor, and the input matching network has been designed to match the input impedance to a $50 \Omega$ source at the operating frequency (36.8 GHz).

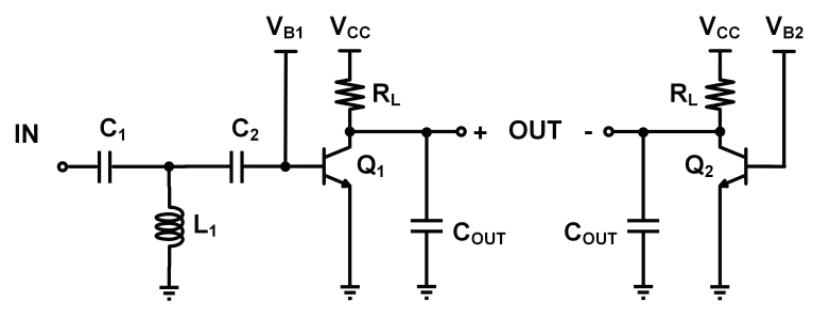

Figure 4. Schematic of the designed CE detector. 
Table 1. Detector Circuit Sizing and Biasing*

\begin{tabular}{c|c|c|c|c}
\hline $\mathrm{V}_{\mathrm{CC}}[\mathrm{V}]$ & $\mathrm{V}_{\mathrm{h}}[\mathrm{V}]$ & $\mathrm{J}_{\mathrm{C}}\left[\mathrm{mA} / \mu \mathrm{m}_{2}\right]$ & $\mathrm{R}_{\mathrm{L}}[\mathrm{k} \Omega]$ & $\mathrm{V}_{\mathrm{b} 1,2}[\mathrm{~V}]$ \\
\hline 2 & 1 & 0.211 & 6.1 & 0.83 \\
$\mathrm{C}_{1}[\mathrm{fF}]$ & $\mathrm{L}_{1}[\mathrm{pH}]$ & $\mathrm{C}_{2}[\mathrm{fF}]$ & $\mathrm{C}_{\mathrm{OUT}}[\mathrm{fF}]$ & $\mathrm{P}_{\mathrm{C}}[\mathrm{mW}]$ \\
39 & 300 & 124 & 250 & 1.26 \\
\hline
\end{tabular}

$* \mathrm{RF}$ input power considered, $\mathrm{P}_{\mathrm{RF}}=-50 \mathrm{dBm}$

Fig. 4 shows the circuit schematic of the designed CE detector. In Table 1 is reported detector's sizing and biasing. The designed CE detector exhibits a considerably high responsivity, low NEP and reasonably low $\mathrm{P}_{\mathrm{C}}$. In fact, the main results of the simulations have shown the following performance: detector's responsivity $\mathfrak{R}_{\text {det }}=300$ $\mathrm{kV} / \mathrm{W}$, detector's output noise equal to $39 \mathrm{nV} \sqrt{ } \mathrm{Hz}$ corresponding to a noise equivalent power NEP $=130 \mathrm{fW} / \sqrt{ } \mathrm{Hz}$.

\subsection{Low-Noise Amplifier}

Given the performance of the detector, presented in the previous subsection, we set the target gain of the LNA to be $>35 \mathrm{~dB}$, while the noise specification is to have $F_{\mathrm{LNA}}$ $<5 \mathrm{~dB}$. The proposed receiver, shown in Fig. 3, has a direct-conversion architecture ${ }^{[17]}$. As such, it is important to have a very-high reverse isolation in the LNA stage. This can be guaranteed by the cascode configuration which also presents a good stability.

Fig. 5 shows the circuit schematic of the designed LNA. It is composed by the cascade of three stages. Each stage is a single-ended cascode amplifier. In Table 2 is reported LNA's sizing and biasing. The first stage is optimized for low-noise, therefore the transistors are biased around minimum noise figure current density. The following stages are optimized for high-gain, therefore the transistors are biased at the maximum $\mathrm{f}_{\mathrm{T}}$ current density. For simultaneous noise and input power matching, we used a general LNA design procedure that takes advantage of the scalability of the devices and of the inductors $\mathrm{L}_{\mathrm{E}}$ and $\mathrm{L}_{\mathrm{B}}$ used for emitter degeneration and input power matching, respectively ${ }^{[40]} . \mathrm{L}_{\mathrm{C}}$ ladders are used for inter-stage matching between stages one and two, and between stages two and three (maximum power transfer). As per the output matching network, the LC ladder used, employs a parasitic resistance to lower the $\mathrm{Q}$ of the inductor $\mathrm{L}_{3}$ and meet the $1 \mathrm{GHz} 3_{\mathrm{dB}}$-bandwidth (BW) specification. It is worth adding that, since the input signal to the radiometric receiver is noise, i.e. very low power levels, linearity is not a requirement and it has not been considered for performance assessment.

As results from the simulations, the performance of the LNA is as follows: LNA's gain and NF at $36.8 \mathrm{GHz}$ are $\mathrm{G}_{\mathrm{LNA}}=38.2 \mathrm{~dB}$ and $\mathrm{NF}=4.0 \mathrm{~dB}$ respectively. To be noted that NF is below $6 \mathrm{~dB}$ at Ka-band. The $\mathrm{S}_{11}$ is $<-30$ $\mathrm{dB}$ around the centre frequency and $<-10 \mathrm{~dB}$ over the entire frequency range. The $\mathrm{S}_{22}$ is $<-20 \mathrm{~dB}$. The power consumption is $9.6 \mathrm{~mW}$.

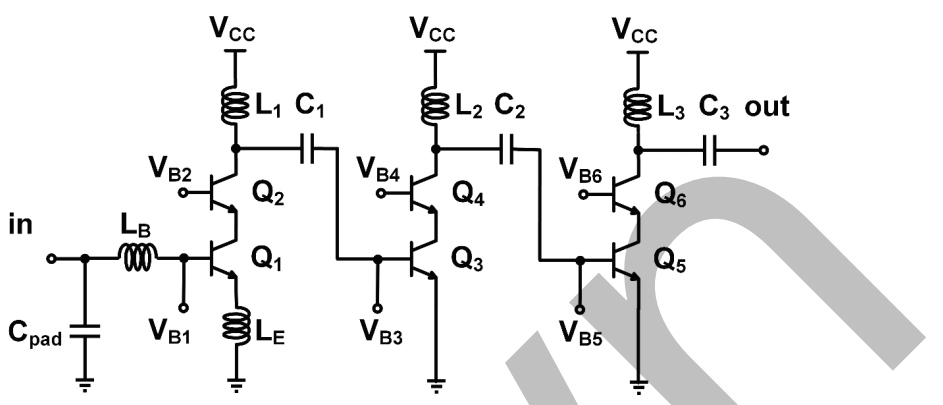

Figure 5. Schematic of the designed LNA

Table 2. LNA Circuit sizing and biasing

\begin{tabular}{c|c|c|c|c|c|c}
\hline $\begin{array}{c}\mathrm{A}_{\mathrm{E} 1,2} \\
{\left[\mu \mathrm{m}^{2}\right]}\end{array}$ & $\mathrm{L}_{\mathrm{B}}[\mathrm{pH}]$ & $\mathrm{L}_{1,2}[\mathrm{pH}]$ & $\mathrm{C}_{1}[\mathrm{fF}]$ & $\mathrm{V}_{\mathrm{b} 1}[\mathrm{~V}]$ & $\mathrm{V}_{\mathrm{b} 3,5}[\mathrm{~V}]$ & $\mathrm{V}_{\mathrm{CC}}[\mathrm{V}]$ \\
\hline 1.2 & 330 & 450 & 23 & 0.82 & 0.85 & 2 \\
$\begin{array}{c}\mathrm{A}_{\mathrm{E} 3.6} \\
{\left[\mu \mathrm{m}^{2}\right]}\end{array}$ & $\mathrm{L}_{\mathrm{E}}[\mathrm{pH}]$ & $\mathrm{L}_{3}[\mathrm{pH}]$ & $\mathrm{C}_{2,3}[\mathrm{fF}]$ & $\mathrm{V}_{\mathrm{b} 2}[\mathrm{~V}]$ & $\mathrm{V}_{\mathrm{b} 4,6}[\mathrm{~V}]$ & $\mathrm{P}_{\mathrm{C}}[\mathrm{mW}]$ \\
0.6 & 160 & 400 & 18 & 1.82 & 1.85 & 9.6 \\
\hline
\end{tabular}

\subsection{Receiver}

The co-integration of the building-blocks to realize the radiometric receiver, will allow improving the performance of both the LNA and the detector, since the parasitics of the interconnections and the matching networks will be minimized when compared to the stand-alone blocks presented in the previous subsections. As a consequence, the performances given for the LNA and detector, can be considered as a worst-case situation. We can calculate the expected responsivity of the co-integrated receiver, as follows:

$$
\mathfrak{R}=\mathfrak{R}_{\text {det }}+10^{G_{L N A} / 20}
$$

which gives, for the simulated results for detector and LNA, a value of $\mathfrak{R}=24 \mathrm{MV} / \mathrm{W}$. Consequently, from (2) and (3), receiver's NEP is about $1.6 \mathrm{fW} / \sqrt{\mathrm{Hz}}$.

\section{Discussion}

The SoC implementation of the radiometer based on the Dicke architecture is now analysed at system level. Referring to Fig. 3 and considering the case of duty cycle $\delta$ $=50 \%$, the input signal to the radiometer is white noise which for half time is collected by the antenna and for half time is provided by the noise generator. In both cases, the signal is modelled as a Gaussian stochastic process having power spectral density (PSD) equal to about $-75 \mathrm{dBm} /$ $\mathrm{Hz}$, corresponding to a noise equivalent temperature of the antenna and of the noise source equal to $1542 \mathrm{~K}$. After the switch, the LNA "shapes" the signal by its transfer function, and adds its inherent noise to it. At the output of the LNA, and at the frequency of $36.8 \mathrm{GHz}$, given the 38 
$\mathrm{dB}$ of gain of the amplifier, the PSD of the signal amounts to about $-37 \mathrm{dBm} / \mathrm{Hz}$. The noise is then processed by the square-law detector, which reveals a voltage proportional to its root mean square value. The synchronous detector (i.e. detector and \pm 1 block) performs also the subtraction between the values in the two switching time intervals. Fig. 6 and Fig. 7 show the evolution of the noise (i.e. the information) through the system in the time and frequency domains. Finally, the integrator extracts the envelope. In our Matlab-Simulink model we implemented the integrator by using an equivalent moving average filter. The output voltage of the integrator for three scenarios of observation is reported in Fig. 8. Therein, quiet Sun refers to the absence of solar flares. The DC voltage at the output can be evaluated as follows ${ }^{[37]}$ :

$$
V_{\text {out }}=k_{B} \Re B W\left(T_{N}-T_{A}\right)
$$

According to (8), quiet Sun corresponds to an output voltage of $0 \mathrm{~V}$; 1000 sfu, i.e. a small entity solar flare, corresponds to $0.32 \mathrm{mV}$; and $1001 \mathrm{sfu}$, corresponds to $0.35 \mathrm{mV}$. Thereby, for an increase of 1 sfu the output voltage increases by $30 \mu \mathrm{V}$. The entire dynamic range from 0 to $150000 \mathrm{sfu}$ (131163 sfu being the strongest flare ever detected at KaBand ${ }^{[29]}$ ) can thus be detected by commercial Analog to Digital Converters (ADCs), as recommended by the ESA ${ }^{[41]}$.

On the basis of the results emerged from our analysis and design, it is finally worth investigating (3) as a function of the integration time $\tau$. Table 3 summarizes the results of LNA, detector and receiver, which we will consider, together with an antenna noise temperature $T_{A}=$ $1542 \mathrm{~K}$ corresponding to the typical value of the quiet Sun (i.e. Sun in absence of flares) at $\mathrm{f}_{0}{ }^{[42]}, \mathrm{BW}=1 \mathrm{GHz}$ and insertion loss (IL) of the switch of $3.5 \mathrm{~dB}^{[25]}$.

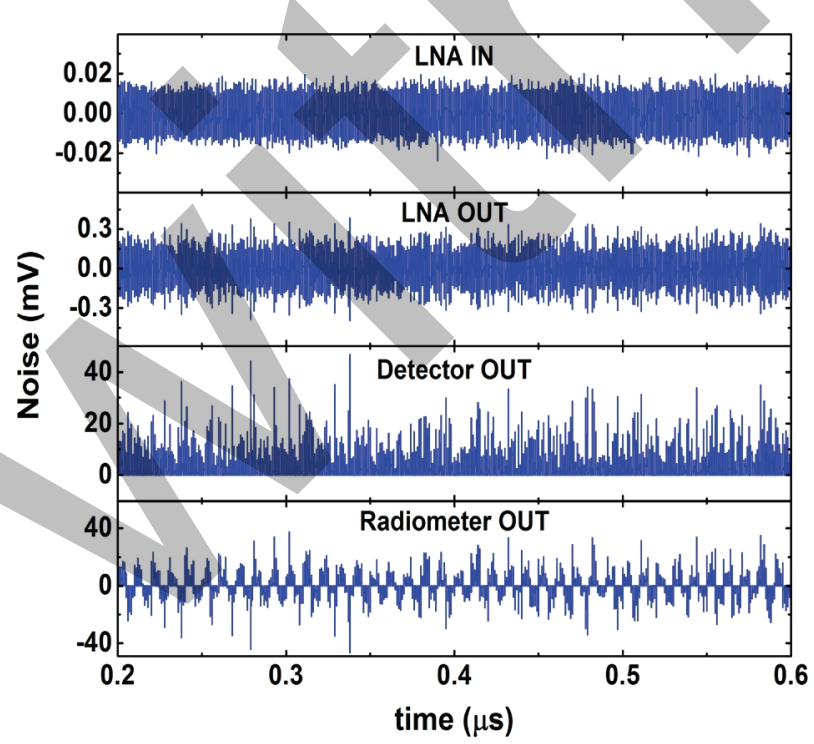

Figure 6. Evolution of the noise signal through the radiometer in the time domain. $\mathrm{T}_{\mathrm{A}}=\mathrm{T}_{\mathrm{N}}=1542 \mathrm{~K}$

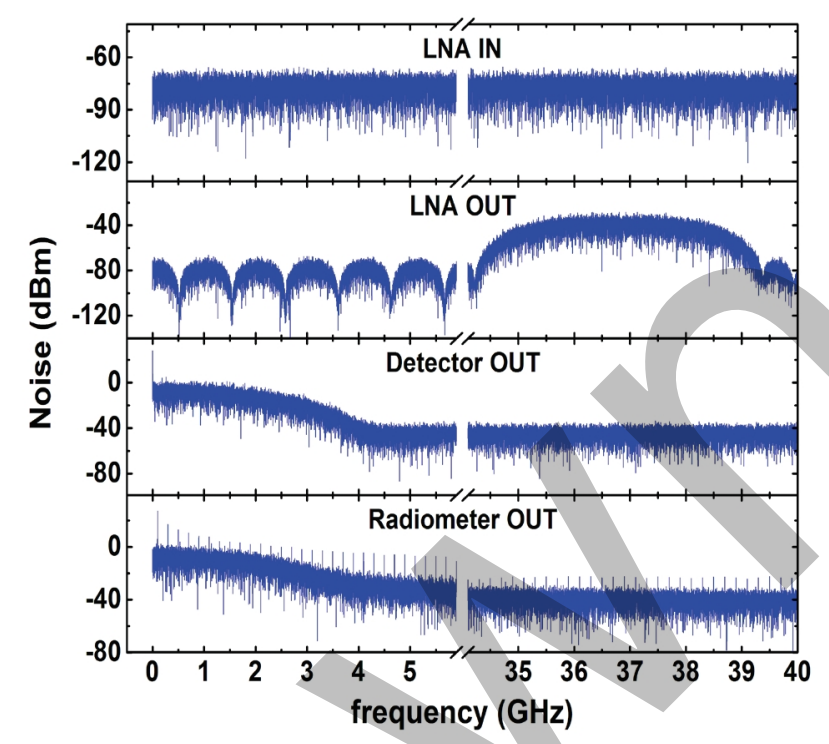

Figure 7. Evolution of the noise signal through the radiometer in the frequency domain. $\mathrm{T}_{\mathrm{A}}=\mathrm{T}_{\mathrm{N}}=1542 \mathrm{~K}$



Figure 8. Voltage at the output of the integrator for three cases: quiet Sun (absence of solar flares) and solar flares of 1000 and $1001 \mathrm{sfu}$

Table 3. Summary of simulation results

\begin{tabular}{c|c|c|c|c|c}
\hline & $\begin{array}{c}\mathrm{G}\left(\mathrm{f}_{0}\right) \\
{[\mathrm{dB}]}\end{array}$ & $\begin{array}{c}\mathrm{NF}\left(\mathrm{f}_{0}\right) \\
{[\mathrm{dB}]}\end{array}$ & $\begin{array}{c}\mathrm{P}_{\mathrm{C}} \\
{[\mathrm{mW}]}\end{array}$ & $\begin{array}{c}\mathrm{NEP}\left(\mathrm{f}_{\mathrm{S}}\right) \\
{[\mathrm{fW} / \sqrt{H z}]}\end{array}$ & {$[\mathrm{MV} / \mathrm{W}]$} \\
\hline $\begin{array}{c}\text { Low-Noise } \\
\text { Amplifier } \\
\text { (LNA) }\end{array}$ & 38.2 & 4.0 & 9.6 & - & - \\
\hline CE-Detector & - & - & 1.3 & 130 & 0.3 \\
\hline Receiver & - & - & 10.9 & 1.6 & 24 \\
\hline
\end{tabular}

The results are shown in Fig. 9. The targeted resolution $\rho s f u$ of $1 \mathrm{sfu}$ is achieved at an integration time $\tau=0.07 \mathrm{~s}$.

It is worth noting that, considering the $0.07 \mathrm{~s}$ integration time and the calculated NEP for the receiver, we have that the weakest detectable signal in presence of noise can be calculated as: 


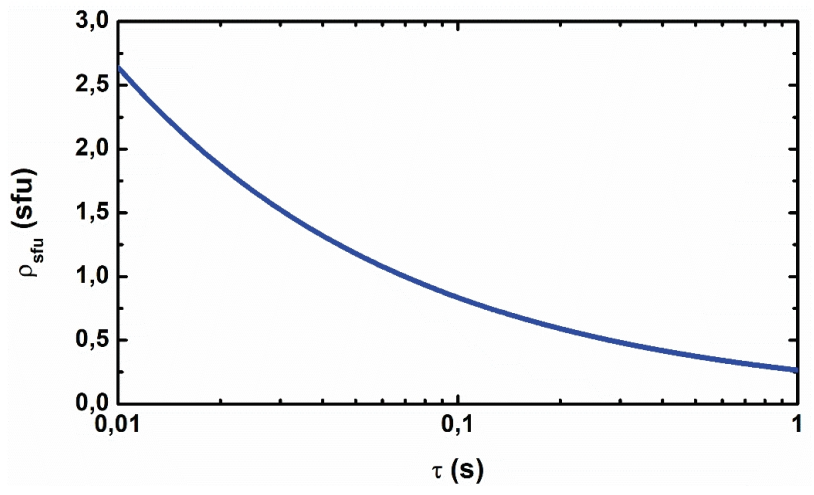

Figure 9. $\rho_{\text {sfu }}$ as a function of the integration time $(\tau)$, from (3)

$$
S_{\text {min }}=\frac{N E P}{\sqrt{2 \tau}}
$$

which gives for the receiver a sensitivity of $2.14 \mathrm{fW}$, corresponding to $-116.7 \mathrm{dBm}$. Given the expected input signal level reported above $(-75 \mathrm{dBm})$, expected noise level is thus about $40 \mathrm{~dB}$ lower than signal level. Table 4 shows a comparison with ground-based instruments.

Table 4. Comparison with ground-based instruments

\begin{tabular}{c|c|c|c|c}
\hline Instrument & $\mathrm{f}_{0}[\mathrm{GHz}]$ & $\mathrm{BW}[\mathrm{GHz}]$ & $\begin{array}{c}\tau \\
{[\mathrm{s}]}\end{array}$ & $\begin{array}{c}\rho \\
{[\mathrm{sfu}]}\end{array}$ \\
\hline NORP $^{[28]}$ & 35 & 1 & 0.1 & 15 \\
Metsahövi $^{[29]}$ & 37 & 1 & 0.02 & 10 \\
This work & 36.8 & 1 & 0.07 & 1 \\
\hline
\end{tabular}

\section{Conclusions}

Solar flares observed in the $\mathrm{mm}$-wave frequency range from a LEO satellite in Heliosynchronous orbit can be detected and measured with a sensitivity much higher than the sensitivity obtained by the current ground-based radiometers. In this paper the activity and results of the EU funded research project FLARES have been presented. In particular, we analyzed the feasibility and design of a system-on-chip Dicke radiometer for space-based detection of solar flares using a commercial space-qualified SiGe technology. Ka-band observations of solar flares as well as the impact of ionospheric fluctuations have been analyzed. The achievable resolution has been derived on the basis of the results of realistic circuit performances estimated for LNA and detector from circuit simulations. A system analysis has also been carried out through Matlab-Simulink simulations. The results show that, in principle, a noise difference equivalent to 1 sfu can be achieved, mostly thanks to the low NEP exhibited by the SiGe detector. This aspect also reduces the complexity of the LNA, so reducing the power consumption requirement.

The general objective of FLARES has been to explore and take a first step towards the SoC approach incorporating analog, mixed-signal and digital integrated circuits, through a commercially available SiGe-BiCMOS technology under space qualification. The state-of-the-art mmwave imagers currently operating in space are based on multi-chip approach, mainly exploiting III-V technologies which allow achieving superior performances but do not allow for integration of all analog and digital subsections. Based on the evaluations emerging from the system analysis, the benefits deriving from SoC integration (miniaturization, reduction of weight, insertion and chip-to-chip losses, power consumption, etc.), the design has been carried out by privileging the reduction of power consumption.

The results obtained from our research demonstrate the high potential and innovation for achieving a significant improvement with respect to the existing ground-based instruments for the detection of solar flares. In particular, the proposed SoC Dicke radiometer can achieve a tentime improvement of resolution, which in turn allows for the detection of solar flares with relatively low intensity, i.e. about 100 times lower than those currently detected by the existing systems, owing to space-borne operations and the microchip-level miniaturization through silicon technology under space qualification. Moreover, innovations such as the miniaturization achievable through SoC radiometers of this kind, and then the possible implementation of on-chip calibrations and on-chip thermal stabilizations, enables significant reductions of weight, size and power consumption, all strategically important for space applications, especially for micro-, nano- and pico-LEO satellite missions.

Finally, it is worth noting that the results of FLARES can be extended to other mm-wave frequency bands, thus providing an optimal basis for further technological developments, especially in terms of 2- and 3-dimensional observations when combined in focal-plane arrays of SoC radiometers.

Acknowledgements: This work has received funding by the EU under the FP7-PEOPLE-2013-IEF FLARES Grant Agreement 625907.

\section{References}

[1] B.T. Tsurutani, O.P. Verkhoglyadova, A.J. Mannucci, G.S. Lakhina, G. Li, and G.P. Zank, "A brief review of solar flare effects on the ionosphere," Radio Science. doi: 10.1029/2008RS004029

[2] K. Shetye, and T. Overbye, "Modeling and analysis of GMD effects on power systems: An overview of the impact on large-scale power systems," IEEE Electrification Magazine, doi: 10.1109/MELE.2015.2480356 
[ 3 ] B. Bala, L.J. Lanzerotti, D.E. Gary, and D.J. Thomson, "Noise in wireless systems produced by solar radio bursts," Radio Science, doi: 10.1029/2001RS002481

[ 4 ] D.D. Morabito, O. P. Verkhoglyadova, D. Han, and J. E. Riedel, "The effects of earthward directed interplanetary coronal mass ejections on near-Earth S band signal links," Radio Science, doi: 10.1029/2011RS004718

[ 5 ] J. León-Tavares, E. Valtaoja, M. Tornikoski, A. Lähteenmäki, and E. Nieppola, "The connection between gamma-ray emission and millimeter flares in Fermi/LAT blazars," A\&A, doi: 10.1051/0004-6361/201116664

[6] System-on-Chip Millimeter-wave Radiometers for Spacebased Detection of Solar Flares. EC-CORDIS. (Online). Available: http://cordis.europa.eu/project/rcn/187972_ en.html

[ 7 ] S. Sahoo, X. Bosch-Lluis, S.C. Reising, and J. Vivekanandan, "Radiometric Information Content for Water Vapor and Temperature Profiling in Clear Skies Between 10 and $200 \mathrm{GHz}$," in IEEE Journal of Selected Topics in Applied Earth Observations and Remote Sensing. doi: 10.1109/ JSTARS.2014.2364394

[ 8 ] T.S. Bastian, A.O. Benz, and D.E. Gary, "Radio emission from solar flares," Annual Review of Astronomy and Astrophysics, doi: 10.1146/annurev.astro.36.1.131

[ 9 ] S. Pojolainen, J. Hildebrandt, M. Karlicky, A. Magun, and I.M. Chertok, "Prolonged millimeter-wave emission from a solar flare near the limb," Astronomy and Astrophysics Journal, doi: 10.1051/0004-6361:20021431

[10] A.B. Tanner, and A. L. Riley, "Design and performance of a high-stability water vapor radiometer," Radio Science, 38(3), 8050, 2003. doi: 10.1029/2002RS002673

[11] R. Villarino et al., "Design and test of the L-band automatic radiometer (LAURA) temperature control," IEEE IGARSS '05., doi: 10.1109/IGARSS.2005.1526773

[12] L. Pazmany and M. Wolde, "A compact airborne G-band $(183 \mathrm{GHz})$ water Vapor Radiometer and retrievals of liquid cloud parameters from coincident radiometer and millimeter wave radar measurements," 2008 Microwave Radiometry and Remote Sensing of the Environment, Firenze, doi: 10.1109/MICRAD.2008.4579473

[13] F. Iturbide-Sanchez, S. C. Reising and S. Padmanabhan, "A Miniaturized Spectrometer Radiometer Based on MMIC Technology for Tropospheric Water Vapor Profiling," in IEEE Transactions on Geoscience and Remote Sensing, doi: 10.1109/TGRS.2007.898444

[14] F. Maiwald et al., "Reliable and Stable Radiometers for Jason-3," in IEEE Journal of Selected Topics in Applied Earth Observations and Remote Sensing, doi: 10.1109/ JSTARS.2016.2535281

[15] L. Colangeli. Announcement of the plans for the issuing of a call for a medium-size mission for launch in 2029-2030
(M5). European Space Agency. [Online]. Available: http:// sci.esa.int/jump.cfm?oid $=56198$

[16] H. Carreno-Luengo, A. Camps; P. Via, J.F. Munoz, A. Cortiella, D. Vidal, J. Jane, N. Catarino, M. Hagenfeldt, P. Palomo, and S. Cornara, "3Cat-2-An Experimental Nanosatellite for GNSS-R Earth Observation: Mission Concept and Analysis," in IEEE Journal of Selected Topics in Applied Earth Observations and Remote Sensing, doi: 10.1109/JSTARS.2016.2574717

[17] L. Aluigi, F. Alimenti, and L. Roselli, "Fully integrated millimeter-wave radiometers: Development level and perspectives," in IEEE RWS, doi: 10.1109/ RWS.2010.5434175

[18] Fonte, D. Zito, and F. Alimenti, "CMOS microwave radiometer: Experiments on down-conversion and direct detections," IEEE ICECS, doi: 10.1109/ICECS.2008.4675092

[19] F. Alimenti, D. Zito, A. Boni, M. Borgarino, A. Fonte, A. Carboni, S. Leone, M. Pifferi, L. Roselli, B. Neri, and R. Menozzi, "System-on-chip microwave radiometer for thermal remote sensing and its application to the forest fire detection," IEEE ICECS, doi: 10.1109/ICECS.2008.4675090

[20] Tomkins, P. Garcia, and S.P. Voinigescu, "A passive W-Band imaging receiver in $65-\mathrm{nm}$ bulk CMOS," IEEE Journal of Solid-State Circuits, doi: 10.1109/ JSSC.2010.2058150

[21] J.W. May, and G.M. Rebeiz, "Design and characterization of W -Band SiGe RFICs for passive millimeter-wave imaging," IEEE Trans. on Microwave Theory and Techniques, doi: 10.1109/TMTT.2010.2042857

[22] L. Gilreath, V. Jain, and P. Heydari, "Design and analysis of a W-Band SiGe direct-detection-based passive imaging receiver," IEEE Journal of Solid-State Circuits, doi: 10.1109/JSSC.2011.2162792

[23] L. Aluigi, T. T. Thai, M. M. Tentzeris, L. Roselli and F. Alimenti, "Chip-to-package wireless power transfer and its application to mm-Wave antennas and monolithic radiometric receivers," 2013 IEEE Radio and Wireless Symposium, Austin, TX, doi: 10.1109/RWS.2013.6486688

[24] L. Aluigi, L. Roselli, S.M. White, and F. Alimenti, "System-on-Chip $36.8 \mathrm{GHz}$ radiometer for space-based observation of solar flares: feasibility study in $0.25 \mathrm{um} \mathrm{SiGe}$ BiCMOS technology," Progress in Electromagnetics Research, doi: 10.2528/PIER12061101

[25] L. Aluigi, F. Alimenti, P. Gallagher, and D. Zito, "Impact of switching on design of Ka-band SoC Dicke radiometer for space detection of solar flares," ISSC, doi: 10.1109/ ISSC.2015.7163776

[26] L. Aluigi, and D. Zito, "Analysis and design of mm-wave detectors in SiGe SoC radiometers for spaceborne observations of solar flares," IEEE Microrad, doi: 10.1109/ MICRORAD.2016.7530502 
[27] L. Aluigi and D. Zito, "Analysis and design of Ka-band SoC radiometer for space detection of solar flares," 2015 IEEE 13th International New Circuits and Systems Conference (NEWCAS), Grenoble, doi: 10.1109/NEWCAS.2015.7182070

[28] NoRP, the Nobeyama Radio Polarimeter, Nobeyama Radio Observatory, Japan. [Online]. Available: http://solar. nro.nao.ac.jp/norp/

[29] Solar Radio Astronomy at Metsähovi, Metsähovi Radio Observatory, Finland. [Online]. Available: http://metsahovi.aalto.fi/en/

[30] F. Berrilli, et al., "The ADAHELI solar mission: investigating the structure of Sun's lower atmosphere," Advances in Space Research, doi: 10.1016/j.asr.2010.01.026

[31] J.A. Ratcliffe, The Magneto-Ionic Theory, Cambridge University Press, United Kingdom, 1959.

[32] S. Chandrasekhar, Radiative Transfer, Dover Publications, USA, 1960.

[33] Y.K. Chasovitin. Typhoon, Obninsk, Russia. [Online]. Available: http://www.wdcb.ru/stp/data

[34] R.S Rawrence, C.G. Little, and H.J.A. Chivers, "A survey of ionospheric effects upon earth-space radio propagation," IEEE Proceedings, doi: 10.1109/PROC.1964.2737

[35] R.H. Dicke, "The measurement of thermal radiation at microwave frequencies," Review of Scientific Instruments, doi: $10.1063 / 1.1770483$
[36] M. Tiuri, "Radio astronomy receivers," IEEE Trans. on Antenna and Propagation, doi: 10.1109/TAP.1964.1138345

[37] L. Aluigi, D. Pepe, F. Alimenti and D. Zito, "K-Band SiGe System-on-Chip Radiometric Receiver for Remote Sensing of the Atmosphere," in IEEE Transactions on Circuits and Systems I: Regular Papers, doi: 10.1109/ TCSI.2017.2761703

[38] IHP, Microelectronics for Aerospace, [Online]. Available: https://www.ihp-microelectronics.com/en/solutions/aerospace.html

[39] Aluigi, L., F. Alimenti, and L. Roselli, "Automatic design and 3D electromagnetic simulation of sub-nH spiral inductors," PIERS Proceedings, 1719-1722, Marrakesh, Morocco, Mar. 20-23, 2011.

[40] L. Aluigi, F. Alimenti and L. Roselli, "Design of a KaBand LNA for SoC space-based millimeter-wave radiometers," 2011 IEEE MTT-S International Microwave Workshop Series on Millimeter Wave Integration Technologies, Sitges, doi: 10.1109/IMWS3.2011.6061863

[41] Bank of Rad-Hard ADCs, MPD 2010. [Online]. Available: http://microelectronics.esa.int/mpd2010/day2/MPD2010 Bank-of-RadHard-ADCs.pdf

[42] H. Nakajima, H. Sekiguchi, M. Sawa, K. Kai, and S. Kawashima, "The radiometer and polarimeter at 80, 35, and $17 \mathrm{GHz}$ for solar observations at Nobeyama," Publ. of the Astronomical Society of Japan, 1985, 37(1), 163-170. 